\title{
Motion Planning for Concentric Tube Robots Using Mechanics-based Models
}

\author{
Luis G. Torres and Ron Alterovitz
}

\begin{abstract}
Concentric tube robots have the potential to enable new minimally invasive surgical procedures by curving around anatomical obstacles to reach difficult-to-reach sites in body cavities. Planning motions for these devices is challenging in part due to their complex kinematics; concentric tube robots are composed of thin, pre-curved, telescoping tubes that can achieve a variety of shapes via extension and rotation of each of their constituent tubes. We introduce a new motion planner to maneuver these devices to clinical targets while minimizing the probability of colliding with anatomical obstacles. Unlike prior planners for these devices, we more accurately model device shape using mechanics-based models that consider torsional interaction between the tubes. We also account for the effects of uncertainty in actuation and predicted device shape. We integrate these models with a sampling-based approach based on the Rapidly-Exploring Roadmap to guarantee finding optimal plans as computation time is allowed to increase. We demonstrate our motion planner in simulation using a variety of evaluation scenarios including an anatomy-based neurosurgery case that requires maneuvering to a difficult-to-reach brain tumor at the skull base.
\end{abstract}

\section{INTRODUCTION}

Concentric tube robots are needle-like devices that can be controlled to trace curved paths through open air [1]-[3]. The ability to control the curvilinear shape of these devices enables them to avoid obstacles and reach targets in tight spaces. These continuum robots are composed of thin nested tubes where each tube is pre-curved, shaped for example as a straight transmission segment followed by a segment with constant curvature. Each tube, typically composed of nitinol, can be controlled independently by two degrees of freedom: it can be (1) rotated axially, and (2) pushed/retracted through the containing tube. These degrees of freedom could be robotically actuated or directly controlled by a human operator. When rotating or telescopically extending any of the component tubes of the robot, the device's entire shape changes due to interactions among the pre-curvatures of the individual tubes.

Paired with effective motion planning algorithms, concentric tube robots have the potential to enable new minimally invasive procedures, such as endoscopic access to the brain [4], the lung [5], [6], and the heart [2]. We illustrate in Fig. 1 the potential of these devices for accessing tumors in the brain near the pituitary gland via the nasal cavity while avoiding critical blood vessels, nerves, and bones.

The power and flexibility of concentric tube robots comes at the cost of modeling and planning complexity. The shape

L. G. Torres and R. Alterovitz are with the Department of Computer Science, University of North Carolina at Chapel Hill, USA luis@cs.unc.edu, ron@cs.unc.edu

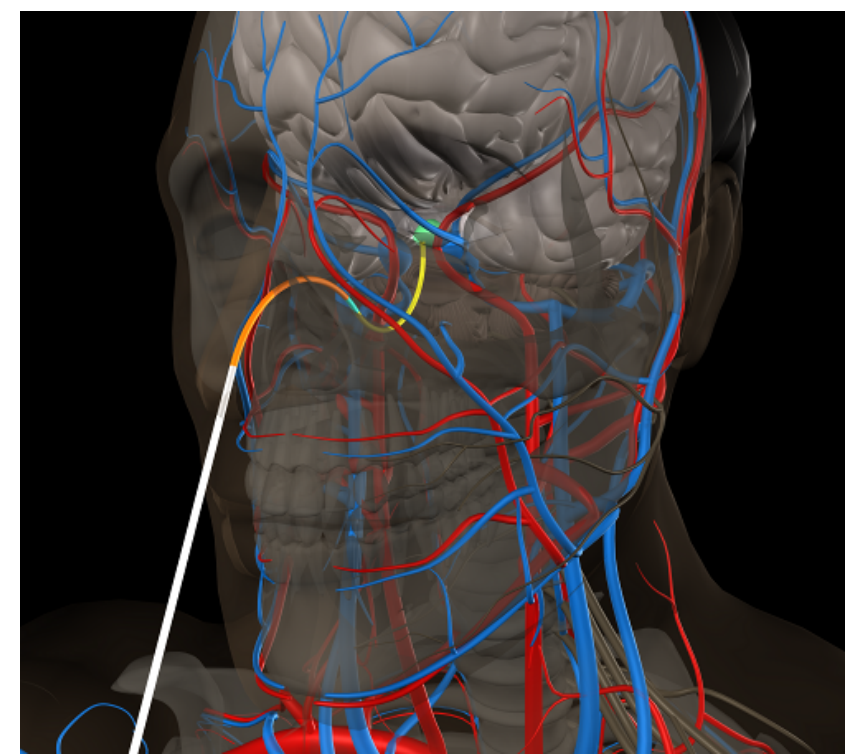

Fig. 1. Virtual simulation of a concentric tube robot executing a motion plan for neurosurgery at the skull base. The robot is inserted through the nostril and guided toward the pituitary gland (highlighted in green) in the skull base while avoiding skin, bone, blood vessels, and healthy brain tissue. The component tubes of the robot are colored gray, orange, cyan, and yellow.

of a concentric tube robot is a function of the control inputs, which are the rotation and extension of each of the component tubes. The shape is governed by the interplay of beam mechanics and torsional effects among the tubes [5], [7]. There is no known closed-form solution to the kinematics of concentric tube robots. Because of these challenges, prior work in motion planning for concentric tube robots in anatomically complex environments has largely focused on simplified kinematic models of the devices at the expense of accuracy. Furthermore, although computationally intensive accurate models of robot shape have been developed, their accuracy typically decreases as the inserted length of the robot increases [8]. In real world scenarios there is uncertainty in actuation and in the kinematic model of the robot which can result in deviations from the predicted motion; this motion uncertainty must be considered in order to maximize the probability of successfully performing a task.

We propose a sampling-based motion planning algorithm that computes the control inputs that guide the robot to a goal point while minimizing the probability of collision with obstacles in the environment. Obstacle avoidance in a minimally-invasive procedure is essential as critical structures like nerves and blood vessels could be damaged if 
contacted by the device. Unlike prior motion planners, our planner uses a highly accurate kinematic model of concentric tube robots. The planner also considers the effect of uncertainty in the estimated shape of the concentric tube robot; using recent results in optimal motion planning, our planner computes plans that minimize the estimated probability of colliding with sensitive structures.

\section{Related Work}

Planning motions for concentric tube robots requires that we have the ability to estimate the robot's shape as a function of its control inputs. Kinematic modeling of concentric tube robots began with geometric and torsionally-rigid models [1], [2], [9]. The model has been improved to consider torsion in straight segments [3], [10], torsion in curved segments [7], [11], and the effect of external loading on the tubes [8], [12]. However, increasing model complexity has also led to longer computation times to estimate the device's shape. In this paper we use a highly accurate model which considers tube bending interactions, tube material properties, and torsional deformation in straight and curved sections of the tubes [11].

Motion planning for concentric tube robots has only addressed special cases of the general problem. Sears et al. formulated the inverse kinematics of concentric tube robots with simplifying physical assumptions [2], [9]. Dupont et al. used a Fourier approximation over a grid of precomputed forward kinematics solutions to approach inverse kinematics for a model of concentric tube robots that fully handles torsional effects on the tubes [7]. Rucker et al. compute inverse kinematics of the externally loaded model of the device by computing the manipulator Jacobian of the robot [13]. These previous approaches do not explicitly consider obstacle avoidance in reaching a target.

Lyons et al. applied optimization-based motion planning in order to find a plan to reach a target point while avoiding obstacles [6], [14] but only considered the simpler kinematic models of concentric tube robots that neglect torsional effects on the tubes. These models allow for the simplifying assumption that any given collision free concentric tube robot configuration can also serve as a collision free motion plan if the appropriate insertion/rotation strategy is used, which enables us to treat the device like a point robot for planning. However, these models sacrifice accuracy for model simplicity. During medical applications, such inaccuracies can lead to significant side effects or procedure failures. The more accurate model we use in this paper invalidates the assumption that inner tube movement does not affect global device shape, which complicates the motion planning process and eliminates the feasibility of using direct configuration optimization methods.

Concentric tube robots with a sufficient number of tubes can be seen as redundant manipulators, which are difficult to plan motions for due to the lack of closed-form inverse kinematics. Weghe et al. and Bertram et al. investigated probabilistic sampling-based approaches in [15], [16] but do not guarantee globally optimal solutions. Recently developed sampling-based algorithms like RRT* [17] can provide probabilistic convergence to an optimal solution; this useful property however is accompanied by a considerable increase in computation time for real-world problems. We base our motion planner on the Rapidly-Exploring Roadmap (RRM) [18] that guarantees probabilistic convergence to the optimal solution but saves computation time by focusing expensive path refinement procedures on paths that can reach the goal region.

Motion planning for a variety of needle-like devices has been addressed for minimally invasive procedures. Optimization-based planning has been used for stiff, symmetric-tip needles in 2D [19] and 3D [20]. Planners have also been developed to maneuver flexible, bevel-tip steerable needles around obstacles in 2D soft tissue [21][26]. However, needles can be approximated as a point robot (i.e. the needle tip) moving through soft tissue since the path of the rest of the needle shaft follows the tip. This approximation does not hold for concentric tube robots operating in open air cavities where robot shape can change globally with tube rotation and extension.

Uncertainty in robot motion is significant in medical applications because of the miniaturization of devices and the complexity of tool/tissue interactions. The effect of uncertainty has been well-studied for bevel-tip steerable needles [22], [23], [26]. The planners described above, however, cannot be directly applied to the case of concentric tube robots. The Stochastic Motion Roadmap presented in [22] assumes a discrete set of possible actions at any given robot state, which is not necessarily true when planning for concentric tube robots. The LQG-based method used in [26] introduces an estimation of the probability of collision during plan execution, but assumes that the robot's tip acts independently of the rest of the shaft. Although we cannot directly use these planners, our goal is to maximize the same metric: the probability of successfully reaching the target while avoiding obstacles.

In this paper we present a motion planning method for concentric tube robots which considers a more accurate kinematic model than in previous work. We present a plan quality metric which seeks to minimize the probability of colliding with obstacles under motion uncertainy. To efficiently compute a plan which optimizes this metric, we extend the RRM with a goal bias while maintaining the RRM's convergence to optimality. Lastly, we demonstrate the effectiveness of our planner by comparing its performance to other sampling-based planners and by generating a motion plan for an anatomy-based neurosurgery procedure.

\section{Problem Formulation}

\section{A. Modeling Assumptions}

We consider a concentric tube robot with $N$ nested tubes numbered in order of decreasing cross-sectional radius. Each tube $i$ consists of a straight transmission segment of length $L_{i}$ followed by a pre-curved portion of length $C_{i}, i=1, \ldots, N$. The pre-curved portions of the tubes are curved with constant radii of curvature of $r_{i}, i=1, \ldots, N$. We assume that the 


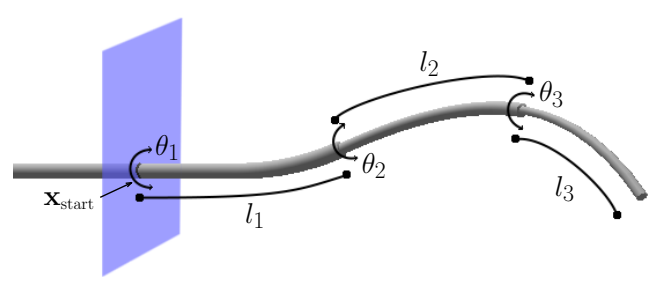

Fig. 2. Configuration $\mathbf{q}=\left(\theta_{1}, l_{1}, \theta_{2}, l_{2}, \theta_{3}, l_{3}\right)$ for a concentric tube robot with $N=3$ nested tubes.

device is inserted at a point $\mathbf{x}_{\text {start }}$ in $3 \mathrm{D}$ space and oriented along the vector $\mathbf{v}_{\text {start }}$.

Each tube contributes two degrees of freedom to the entire concentric tube robot, as shown in Fig. 2. Each tube may be (1) inserted or retracted from the previous tube, and (2) axially rotated. We therefore define a configuration of a concentric tube robot as a $2 N$ dimensional vector $\mathbf{q}=\left(\theta_{i}, l_{i}\right.$ : $i=1, \ldots, N)$ where $\theta_{i}$ is the axial angle at the base of the $i$ 'th tube and $l_{i}>0$ is the length of insertion of tube $i$ past the tube just before it (and $l_{1}$ is the length of the first tube past the insertion point $\mathbf{x}_{\text {start }}$ ).

For a given configuration $\mathbf{q}_{i}$, we define the device's shape using $\mathbf{x}(\mathbf{q}, s): \mathbb{R}^{2 N} \times \mathbb{R} \mapsto \mathbb{R}^{3}$. The function $\mathbf{x}$ is a $3 \mathrm{D}$ space curve parameterized by arc length $s$ in the domain $[0, t]$, where $t$ is the arc length of the innermost tube past the insertion point (equivalent to $\left.\sum_{i} l_{i}\right)$. We note $\mathbf{x}(\mathbf{q}, 0)$ maps to $\mathbf{x}_{\text {start }}$ and $\mathbf{x}(\mathbf{q}, t)$ maps to the $3 \mathrm{D}$ position of the tip of the concentric tube robot. The space curve $\mathbf{x}$ defines only the centerline of the backbone shape of the concentric tube robot. We also define $R(\mathbf{q}, s)$ as the cross-sectional radius of the tube at arc length $s$. To estimate the device's shape $\mathbf{x}(\mathbf{q}, s)$ we use the mechanics-based model developed by Rucker et al. [11].

We define environmental obstacles using a set $O$ of size $M, O=O_{1}, O_{2}, \ldots, O_{M}$. Obstacles can be defined in any geometric representation, as long as it is possible to compute $d\left(\mathbf{x}, O_{j}\right)$, the Euclidean distance between a point $\mathbf{x}$ in space and the obstacle $O_{j}$. In our work we consider spherical obstacles as well as obstacles defined by general 3D polygonal meshes.

\section{B. Planning Problem Formulation}

We define a path $\Pi$ in configuration space as a sequence of robot configurations $\left(\mathbf{q}_{1}, \mathbf{q}_{2}, \ldots, \mathbf{q}_{n}\right)$. A collision free path is a path where, for $i=1, \ldots, n$, the shape of the robot corresponding to $\mathbf{q}_{i}$ does not collide with any obstacles in the environment.

We desire that the robot tip reach a point within a distance of $r_{\text {tol }}$ of a goal point $\mathbf{x}_{\text {goal }}$. We assume that the start location $\mathbf{x}_{\text {start }}$ and initial orientation $\mathbf{v}_{\text {start }}$ are fixed and correspond to the opening through which the device may be deployed based on the constraints of the procedure. From the start location and orientation, we can trivially determine the start configuration $\mathbf{q}_{1}$.

Given the concentric tube robot's properties, the start configuration $\mathbf{q}_{1}$, the goal coordinate $\mathbf{x}_{\text {goal }}$, and the set of obstacles $O$, we formulate the motion planning problem as a search for a path $\Pi=\left(\mathbf{q}_{1}, \ldots, \mathbf{q}_{n}\right)$ where:

1) $\left\|\mathbf{x}\left(\mathbf{q}_{n}, t\right)-\mathbf{x}_{\text {goal }}\right\| \leq r_{\text {tol }}$;

2) Each configuration along $\Pi$ is collision free;

3) $\Pi$ is of maximal quality as defined in the next subsection.

\section{Quantifying Plan Quality}

Our motion planning method allows us to select optimal plans based on any quality metric that is smooth and can be represented as a summation along transitions between configurations in $\Pi$. The choice of metric should ultimately depend on the clinical task. In prior work, we considered minimizing control effort as defined by the total Euclidean distance in configuration space traveled by the path [18].

When planning motions for a physical implementation of a robot, uncertainty in control inputs and in the kinematic model of the robot can cause deviations from the predicted shape. During plan execution, errors in predicted shape can accumulate and possibly lead to the failure of a plan to be collision free or to reach the goal. In a medical procedure, obstacle collisions can result in significant harm to the patient, e.g. a vital blood vessel may be punctured by the robot. We therefore consider a metric that minimizes the estimated probability of colliding with sensitive structures.

For a given path $\Pi=\left(\mathbf{q}_{1}, \ldots, \mathbf{q}_{n}\right)$, we define its probability of clearance $P_{\text {clear }}(\Pi)$ as the probability that all of its configurations $\mathbf{q}_{i}$ are collision free. We denote the probability of configuration $\mathbf{q}_{i}$ being collision free as $P_{\text {clear }}\left(\mathbf{q}_{i}\right)$.

For a given robot configuration $\mathbf{q}_{i}$, we characterize its probability of being collision free as the probability that the shape of the concentric tube robot at configuration $\mathbf{q}_{i}$ is free of collision with any of the obstacles $O_{j}, j=1, \ldots, M$. A configuration $\mathbf{q}_{i}$ is collision free only if for every 3D point $\mathbf{x}(s)$ on the space curve defining the robot's shape and for every obstacle $O_{j}$, the distance between $\mathbf{x}(s)$ and $O_{j}$ is greater than the radius of the concentric tube robot at $s$. We discuss in the next section how the planner estimates the probability of clearance for a configuration $\mathbf{q}$.

\section{Motion Planning Algorithm}

We use a sampling-based approach to solve the motion planning problem formulated in Sec. III-B. Our planner combines probabilistic completeness for finding a path that avoids obstacles with the property that it converges to the optimal solution (under a metric such as minimizing control effort or maximizing estimated probability of clearance) as computation time is allowed to increase.

\section{A. Rapidly-Exploring Roadmaps}

Our motion planner is based on the Rapidly-Exploring Roadmaps method [18] that provides both probabilistic completeness and convergence to optimality guarantees while efficiently only optimizing paths that can feasibly reach the goal. The RRM algorithm generates a weighted, directed graph whose vertices represent robot configurations and weighted edges correspond to the cost associated with 
moving from one configuration to another. The algorithm begins with a vertex at the start configuration $\mathbf{q}_{1}$. It expands in an RRT-like manner by adding vertices and edges toward randomly sampled points in the configuration space. Once there exists at least one sequence of connected vertices from the start vertex to the goal region, the RRM then at each iteration either continues random exploration or refines an existing path to the goal to obtain a better solution. The userspecified parameter $w_{\text {refine }} \in[0,1)$ weighs these two options; the RRM algorithm will explore the configuration space with probability $\left(1-w_{\text {refine }}\right)$ and will refine with probability $1-w_{\text {refine }}$. The algorithm avoids wasteful refinement by only refining paths that can feasibly reach the goal. Once the algorithm has run for a certain number of iterations, then the method uses Dijsktra's shortest path algorithm to find the path with lowest total cost from the start configuration to a goal configuration.

\section{B. Goal Bias}

To reduce planning computation time, we extend the RRM method by introducing a goal bias. Goal bias can significantly speed up sampling based planners by checking for connections to the goal rather than requiring that a configuration be sampled inside the goal region. To introduce a goal bias into RRM, we define another weighting parameter $w_{\text {goal }}$ so that, at each iteration of the RRM algorithm, we have the choice of the following actions:

1) Explore: Rapidly explore with probability $\left(1-w_{\text {refine }}-\right.$ $\left.w_{\text {goal }}\right)$.

2) Goal bias: Check for a connection to the goal with probability $w_{\text {goal }}$.

3) Refine: Refine a feasible path with probability $w_{\text {refine }}$ To perform a goal biasing step, we need to generate configurations that result in the robot tip position $\mathbf{x}(\mathbf{q}, t)$ being inside the goal region around $\mathbf{x}_{\text {goal }}$. This is an inverse kinematics problem, and it is made difficult because there is no known closed-form solution to the kinematics of concentric tube robots (using accurate beam mechanics models and torsion). We can however utilize the robot's manipulator Jacobian to approximate a change in configuration inputs that will result in the robot's tip moving closer to the goal. The manipulator Jacobian relates a differential change in the robot's configuration to a differential change in the position of the end effector of the robot.

At each goal biasing step we select the vertex $\mathbf{q}_{\text {near }}$ in the RRM that has a tip position closest to $\mathbf{x}_{\text {goal }}$ and was not previously selected or created as part of a goal bias operation. We define our desired change in robot tip position to be $\Delta \mathbf{x}_{\text {tip }}=\mathbf{x}_{\text {goal }}-\mathbf{x}\left(\mathbf{q}_{\text {near }}, t\right)$. We then compute the manipulator Jacobian $J\left(\mathbf{q}_{\text {near }}\right)$ using a second-order finite difference approximation. By taking the pseudoinverse of the Jacobian $J^{+}$we can then get a linear approximation of the required change in configuration $\Delta \mathbf{q}$ which will bring the tip of the robot closer to the goal $\left(\Delta \mathbf{q} \approx J^{+} \Delta \mathbf{x}_{\text {tip }}\right)$. At each goal biasing step we iteratively apply this operation, taking small steps in configuration space and adding these intermediate configurations to the RRM graph until we either collide with an obstacle or stop making positive progress toward the goal. Our approach to the goal bias is similar to that of the JT-RRT algorithm [15], with the exception that we use pseudoinverses of the Jacobian instead of transposes for better performance with concentric tube robots.

\section{Computing Path Cost}

As discussed in Sec. III-C, our motion planning method can support a variety of metrics for quantifying the quality of paths. We would like to minimize the probability that this uncertainty results in collision with obstacles.

We first estimate the probability of a given robot configuration being collision free. For a given robot configuration $\mathbf{q}$ we can calculate the shape of the robot $\mathbf{x}(\mathbf{q}, s)$ parameterized by arc length $s$. We discretize this space curve into a finite sequence of $3 \mathrm{D}$ points $\left(\mathbf{x}_{1}, \ldots, \mathbf{x}_{n}\right)$ such that $\mathbf{x}_{1}=\mathbf{x}(\mathbf{q}, 0)$ and $\mathbf{x}_{n}=\mathbf{x}(\mathbf{q}, t)$, i.e. $\mathbf{x}_{1}$ corresponds to the insertion point $\mathbf{x}_{\text {start }}$ and $\mathbf{x}_{n}$ corresponds to the position of the tip of the robot. Uncertainty in the positions of these points $\mathbf{x}_{i}$ as a result of the factors previously described create a non-zero probability of colliding with an obstacle and causing a failure in the procedure. We model this uncertainty as a normal distribution about each $\mathbf{x}_{i}$ 's position in 3D space. As inspired by [27], we define the probability of $\mathbf{x}_{i}$ being in collision as the likelihood of its 3D position varying by more than its distance from the closest obstacle in the environment. We therefore approximate the probability of a robot configuration $\mathbf{q}$ being collision free as

$$
P_{\text {clear }}(\mathbf{q})=\prod_{i}\left[P_{\text {clear }}\left(\mathbf{x}_{i}\right)\right]^{\frac{1}{n}}
$$

where the probability $P_{\text {clear }}\left(\mathbf{x}_{i}\right)$ of a point $\mathbf{x}_{i}$ being collision free is estimated by a chi distribution of standard deviation $\sigma(s)$ about $\mathbf{x}_{i}$ 's distance from the nearest obstacle. We take a geometric mean in (1) so as to make the probability invariant to the discretization resolution of the robot's shape. The standard deviation $\sigma(s)$ is dependent on the arc length parameter of $\mathbf{x}_{i}$ because uncertainty in the shape of the robot typically increases with the arc length along the robot toward the tip [8].

We experimentally characterized $\sigma(s)$ by executing randomized motion plans with Gaussian noise in actuator inputs, material properties of the tubes, and orientation of tubes as they exit outer tubes. The variation in $3 \mathrm{D}$ position due to noise of each point $\mathbf{x}_{i}$ on the cannula shaft was collected. We fit the experimental data to a chi distribution using the parameter estimation method in [28].

Lastly, we need to restate our problem of maximizing the probability of clearance of a path in terms of minimizing the summation of edge costs in the RRM graph. The estimated probability of clearance for an entire path, $P_{\text {clear }}(\Pi)$, can be computed as the product of the $P_{\text {clear }}\left(\mathbf{q}_{i}\right)$ for all configurations $\mathbf{q}_{i}$ in path $\Pi$. To express this product as a summation we use a negative log transformation; we therefore assign each edge cost as

$$
E\left(\mathbf{q}_{i}, \mathbf{q}_{j}\right)=-\log \left[P_{\text {clear }}\left(\mathbf{q}_{j}\right)\right]
$$


where $E\left(\mathbf{q}_{i}, \mathbf{q}_{j}\right)$ is the weight of the edge connecting configurations $\mathbf{q}_{i}$ and $\mathbf{q}_{j}$. With edge weights defined this way, graph paths with smaller edge weights correspond to paths with a higher estimated probability of clearance.

\section{Convergence to Optimality}

Our planning algorithm always converges in the limit to a globally optimal solution if one exists. In [18] it is shown that a RRM will always converge to an optimal solution in the limit as long as the RRM executes path refinement at least as often as it randomly explores the configuration space. We will now show that, under certain conditions, we can still meet this convergence property with the incorporation of the goal bias that we added in this paper.

The RRM algorithm keeps a list of vertices that still require refinement, $U$. Convergence to a globally optimal solution requires that $|U|$ does not grow unbounded as the number of iterations executed approaches infinity. In the standard algorithm, at each iteration the RRM can either explore and add a node to $U$ or refine and remove a node from $U$. With the addition of a goal biasing step, the RRM has an additional choice of extending toward the goal and adding up to a specified maximum number of nodes. Depending on the likelihood of the RRM to randomly explore, perform a goal biasing step, or perform refinement, we can find a maximum number of nodes to allow the RRM to add at each goal biasing step to maintain the convergence to global optimality.

Let $X_{k}$ be the number of vertices added to $U$ and $U_{k}$ be the total number of vertices in $U$ at the $k^{\prime}$ th iteration of the RRM. As previously defined, the probabilities of refining, goal bias, and exploration as $w_{\text {refine }}, w_{\text {goal }}$, and $\left(1-w_{\text {refine }}-w_{\text {goal }}\right)$, respectively. Given a maximum number of nodes added during the goal bias step, $G$, we can calculate the expected values of $X_{k}$ and $U_{k}$ at the $k^{\prime}$ th iteration of the RRM:

$$
\begin{aligned}
E\left(X_{i}\right) & =\left(1-w_{\text {refine }}-w_{\text {goal }}\right)+w_{\text {goal }} G-w_{\text {refine }} \\
E\left(U_{k}\right) & =1+E\left(X_{1}+X_{2}+\ldots+X_{k}\right)=1+\sum_{i} E\left(X_{i}\right) \\
& =1-2 k w_{\text {refine }}+k w_{\text {goal }}(G-1)+k
\end{aligned}
$$

Because we are interested in determining the maximum number of vertices $G$ to add at each goal biasing step to make $U$ empty in the limit, we take the limit of $E\left(U_{k}\right)$ as $k$ approaches infinity, set it to zero, and solve for $G$ :

$$
G=\frac{2 w_{\text {refine }}-1}{w_{\text {goal }}}+1
$$

Therefore, given values for $w_{\text {refine }}$ and $w_{\text {goal }}$, we can use (4) to calculate the maximum number of nodes to add during a goal biasing iteration while still maintaining the theoretical convergence to optimality afforded by the RRM.

\section{Results}

For our experiments we simulated a concentric tube robot with the following tube parameters: straight tube section lengths $L_{i}$ of $12.5 \mathrm{~cm}, 25 \mathrm{~cm}$, and $37.5 \mathrm{~cm}$; pre-curved section lengths $C_{i}$ of $5 \mathrm{~cm}, 10 \mathrm{~cm}$, and $15 \mathrm{~cm}$; cross-sectional

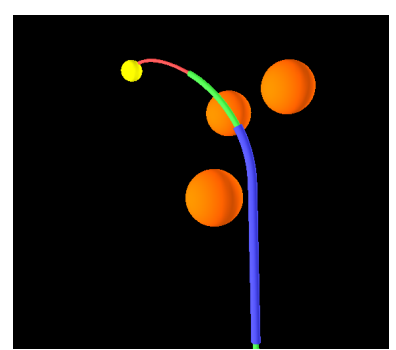

(a)

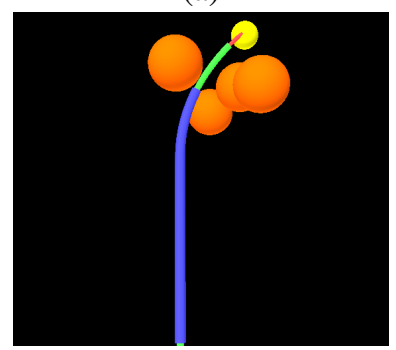

(c)

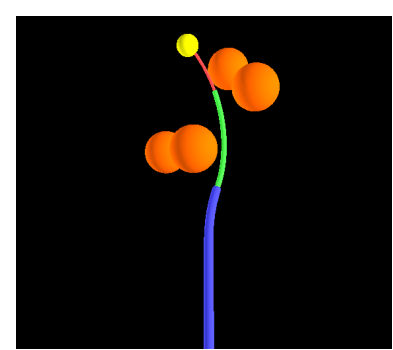

(b)

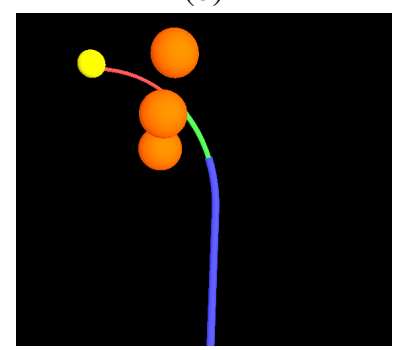

(d)
Fig. 3. Four test environments, each containing spherical obstacles.

radii $R_{i}$ of $0.125 \mathrm{~cm}, 0.0875 \mathrm{~cm}$, and $0.05 \mathrm{~cm}$; and radii of curvature $r_{i}$ for all pre-curved sections of $10 \mathrm{~cm}$. The material properties of the tubes were defined to be equivalent to those of nitinol. The growth of the $\sigma(s)$ function described in Sec. IV-C was found to be linear with a coefficient of 0.03559 .

\section{A. Spherical Obstacle Environment}

In our first set of experiments we generated four environments with a goal region and arbitrarily dispersed spherical obstacles to obstruct the motion of the simulated concentric tube robot. The four environments are demonstrated in Fig. 3. In each experiment we ran our planner for 10,000 iterations using $w_{\text {explore }}=0.7$ and $w_{\text {goal }}=0.01$ (i.e. we performed random exploration $70 \%$ of the time, biased toward the goal $1 \%$ of the time, and performed refinement $29 \%$ of the time). For comparison, we also ran an RRT planner and an RRG planner [17]. The RRT reflects a planner with no refinement and the RRG eagerly performs all possible refinement at each iteration.

As long as we employed a goal bias, our planner never failed to find a collision free path to the goal in environments where such a path was known to exist. We also ran experiments using a planner without a goal bias and even after upwards of 60,000 iterations a path was never found for any of the environments; therefore our incorporation of a goal bias was essential to the effectiveness of our planner. We show a comparison of the run times for RRT, RRG, and RRM in Fig. 4.

To verify the correlation of our estimated $P_{\text {clear }}$ metric with the actual probability of clearance of a plan under uncertainty, we executed each of the generated plans 100 times with small amounts of Gaussian noise in the kinematic model computations to test the plans' robustness to uncertainty. A run was considered "clear" if the robot did not collide into any obstacles. For comparison, we also ran the above 


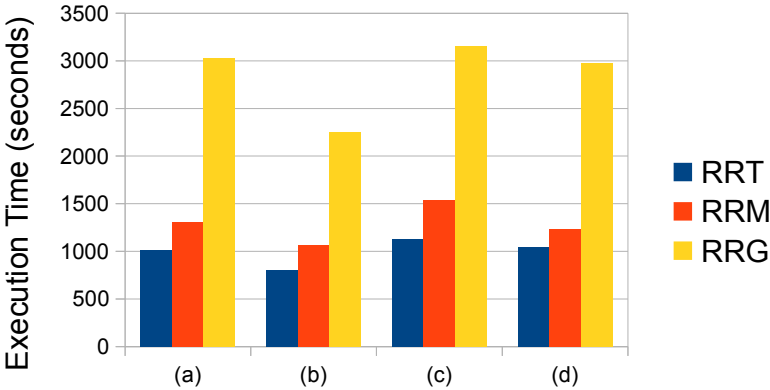

Environment

Fig. 4. Execution times in the test environments shown in Fig. 3 for each evaluated planning algorithm. We also note that the quality metric values of plans generated by the RRM were always better than RRT plans and always within $1 \%$ of the RRG plans.

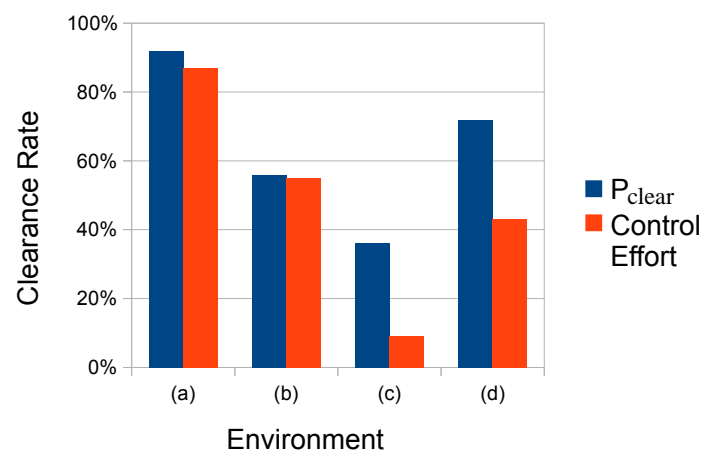

Fig. 5. Clearance rates of Monte Carlo simulations of plans computed for the four test environments with spherical obstacles. Plans were a result of 10,000 iterations of our algorithm using both the $P_{\text {clear }}$ metric and a minimal control effort metric.

experiments on a set of plans generated by a metric that minimizes control effort. In Fig. 5, we compare the clearance rates obtained by minimizing control effort to the clearance rates of plans generated using the $P_{\text {clear }}$ metric. The clearance rate for plans computed using the $P_{\text {clear }}$ metric was higher than that of the plans that minimized control effort.

\section{B. Neurosurgery Environment}

Tumors located at the skull base (in or near the pituitary gland) are difficult to safely access, making surgical removal a challenge. Rather than cutting through healthy brain tissue to access these tumors, a preferred route is through the nasal cavity (an endonasal approach). However, current surgical tools provide limited ability to access sites at the skull base. Concentric tube robots have the potential to help physicians performing these procedures by entering a nostril, curving through the nasal cavity, drilling a small hole through bone at the skull base, and then entering the tumor site in the brain while avoiding critical nerves and blood vessels.

We evaluate our planner in a realistic anatomical environment that includes the skull, critical blood vessels, and healthy brain tissue. In our planner implementation, we use PQP [29] for distance queries between our concentric tube robot and obstacles in the anatomical environment. The planner required 1077 seconds of computation time. As shown in Fig. 6, the plan cleanly avoids bone, critical blood vessels, and healthy brain tissue en route to the skull base tumor. With the dexterity of concentric tube robots and guidance by our motion planner, we can find a path that reaches the tumor while minimizing the probability of damaging vital structures during execution.

\section{Conclusion}

We proposed a motion planning algorithm for guiding concentric tube robots around obstacles to goal regions. Our algorithm is based on Rapidly-Exploring Roadmaps (RRM) and a mechanically accurate kinematic model of concentric tube robots. Given a goal location, the starting position and orientation, and a geometric representation of the environmental obstacles, our algorithm computes a sequence of rotation angles and insertion lengths for each tube of the concentric tube robot such that the device follows a collision free path to the goal. The path computed by our planner probabilistically converges to a globally optimal solution. We presented a metric of path optimality which is based on the probability of safely avoiding obstacles during plan execution. In this way we compute plans which are more robust to uncertainty in the predicted shape of the concentric tube robot compared to plans computed by a standard metric that minimizes control effort.

In future work, we will investigate new difficult surgical procedures that would benefit from the dexterity and minimal invasiveness of concentric tube robots. Finally, we plan to integrate our motion planner with concentric tube robot hardware and image-guided sensing to fully evaluate the exciting potential of these devices with integrated motion planning software.

\section{AcKNowledGeMENTS}

The authors thank Robert J. Webster III and Caleb Rucker from Vanderbilt University for their insights regarding concentric tube robots, Dr. Matthew G. Ewend and Dr. Anand Germanwala from the Department of Neurosurgery at UNCChapel Hill for their feedback on the clinical application of concentric tube robots to skull base surgery, and Nathan Dierk for creating visualizations of these devices. This research was supported in part by the National Science Foundation (NSF) through an NSF Graduate Research Fellowship and grant \#IIS-0905344 and by the National Institutes of Health (NIH) under grant \#R21EB011628.

\section{REFERENCES}

[1] J. Furusho, T. Katsuragi, T. Kikuchi, T. Suzuki, H. Tanaka, Y. Chiba, and H. Horio, "Curved multi-tube systems for fetal blood sampling and treatments of organs like brain and breast," J. Comput. Assist. Radiol. Surg., vol. 1, pp. 223-226, 2006.

[2] P. Sears and P. Dupont, "A steerable needle technology using curved concentric tubes," in Proc. IEEE/RSJ Int. Conf. on Intelligent Robots and Systems (IROS), Oct. 2006, pp. 2850-2856. 

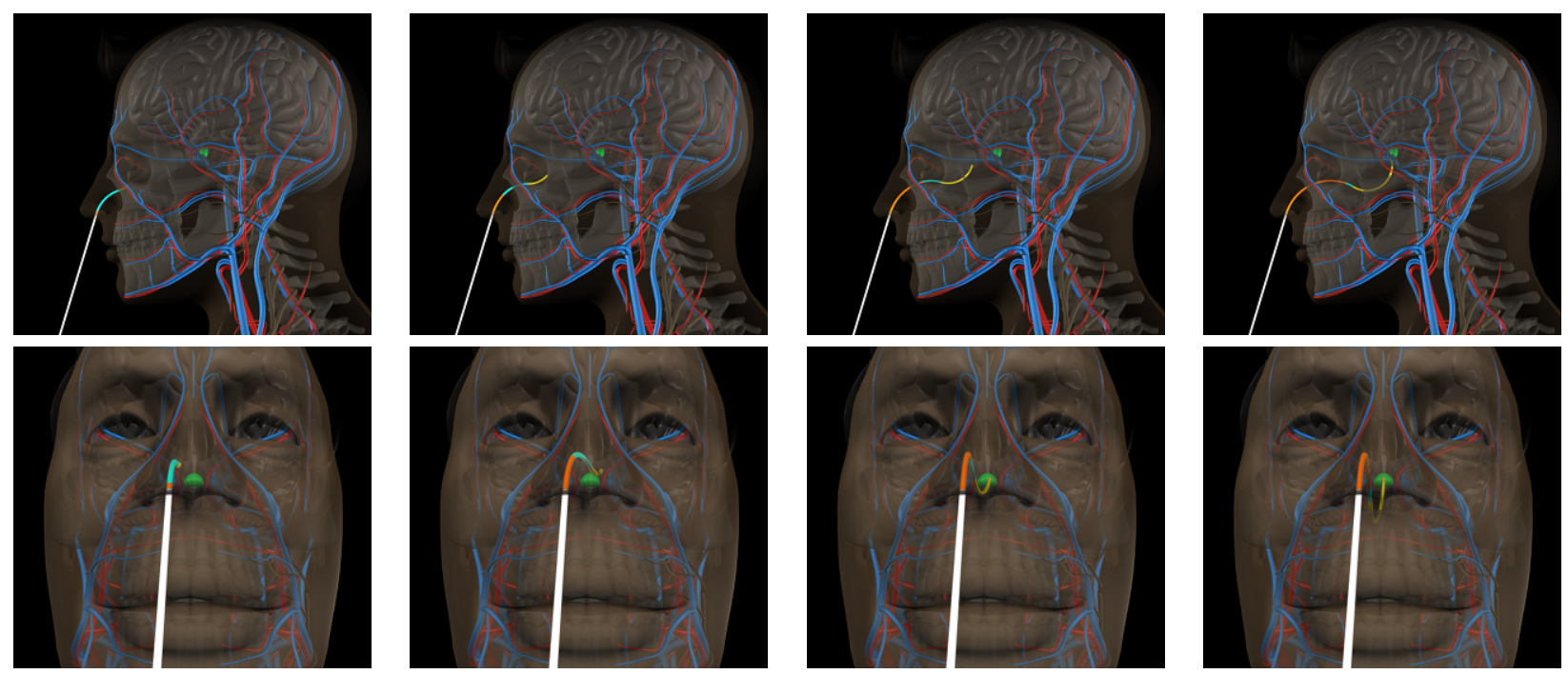

Fig. 6. A step-by-step illustration of our simulation of the minimally invasive procedure discussed in V-B. The concentric tube robot is inserted based on a motion plan that reaches a clinical target at the skull base while avoiding obstacles including skin, bone, blood vessels, and healthy brain tissue. The same procedure is shown from two perspectives.

[3] R. J. Webster III, A. M. Okamura, and N. J. Cowan, "Toward active cannulas: Miniature snake-like surgical robots," in Proc. IEEE/RSJ Int. Conf. on Intelligent Robots and Systems (IROS), 2006, pp. 2857-2863.

[4] A. J. Martin, W. A. Hall, C. Roark, P. A. Starr, P. S. Larson, and C. L. Truwit, "Minimally invasive precision brain access using prospective stereotaxy and a trajectory guide." J Magn Reson Imaging, vol. 27, no. 4, pp. 737-743, Apr 2008.

[5] R. J. Webster III, J. M. Romano, and N. J. Cowan, "Mechanics of precurved-tube continuum robots," IEEE Trans. Robotics, vol. 25, no. 1, pp. 67-78, Feb. 2009.

[6] L. A. Lyons, R. J. Webster III, and R. Alterovitz, "Planning active cannula configurations through tubular anatomy," in Proc. IEEE Int. Conf. Robotics and Automation (ICRA), May 2010, pp. 2082-2087.

[7] P. E. Dupont, J. Lock, B. Itkowitz, and E. Butler, "Design and control of concentric-tube robots," IEEE Trans. Robotics, vol. 26, no. 2, pp. 209-225, Apr. 2010

[8] D. C. Rucker, B. A. Jones, and R. J. Webster III, "A geometrically exact model for externally loaded concentric-tube continuum robots," IEEE Trans. Robotics, vol. 26, no. 5, pp. 769-780, Oct. 2010.

[9] P. Sears and P. E. Dupont, "Inverse kinematics of concentric tube steerable needles," in Proc. IEEE Int. Conf. Robotics and Automation (ICRA), Apr. 2007, pp. 1887-1892.

[10] P. E. Dupont, J. Lock, and E. Butler, "Torsional kinematic model for concentric tube robots," in Proc. IEEE Int. Conf. Robotics and Automation (ICRA), May 2009, pp. 3851-3858.

[11] D. C. Rucker and R. J. Webster III, "Parsimonious evaluation of concentric-tube continuum robot equilibrium conformation," IEEE Trans. Biomedical Engineering, vol. 56, no. 9, pp. 2308-2311, Sep. 2009.

[12] J. Lock, G. Laing, M. Mahvash, and P. E. Dupont, "Quasistatic modeling of concentric tube robots with external loads," in Proc. IEEE/RSJ Int. Conf. on Intelligent Robots and Systems (IROS), Oct. 2010, pp. 2325-2332.

[13] D. C. Rucker and R. J. Webster III, "Computing Jacobians and compliance matrices for externally loaded continuum robots," in Proc. IEEE Int. Conf. Robotics and Automation (ICRA), May 2011.

[14] L. A. Lyons, R. J. Webster III, and R. Alterovitz, "Motion planning for active cannulas," in Proc. IEEE/RSJ Int. Conf. on Intelligent Robots and Systems (IROS), Oct. 2009, pp. 801-806.

[15] M. V. Weghe, D. Ferguson, and S. Srinivasa, "Randomized path planning for redundant manipulators without inverse kinematics," in Proc. IEEE Int. Conf. Robotics and Automation (ICRA), Dec. 2007, pp. $477-482$

[16] D. Bertram, J. Kuffner, R. Dillmann, and T. Asfour, "An integrated approach to inverse kinematics and path planning for redundant manipulators," in Proc. IEEE Int. Conf. Robotics and Automation (ICRA), May 2006, pp. 1874-1879.
[17] S. Karaman and E. Frazzoli, "Incremental sampling-based algorithms for optimal motion planning," in Proc. Robotics: Science and Systems, 2010.

[18] R. Alterovitz, S. Patil, and A. Derbakova, "Rapidly-exploring roadmaps: Weighing exploration vs. refinement in optimal motion planning," in Proc. IEEE Int. Conf. Robotics and Automation (ICRA), May 2011, pp. 3706-3712.

[19] R. Alterovitz, J. Pouliot, R. Taschereau, I.-C. Hsu, and K. Goldberg, "Sensorless planning for medical needle insertion procedures," in Proc. IEEE/RSJ Int. Conf. on Intelligent Robots and Systems (IROS), Oct. 2003, pp. 3337-3343.

[20] E. Dehghan and S. E. Salcudean, "Needle insertion point and orientation optimization in non-linear tissue with application to brachytherapy," in Proc. IEEE Int. Conf. Robotics and Automation (ICRA), Apr. 2007, pp. 2267-2272.

[21] R. Alterovitz, K. Goldberg, and A. M. Okamura, "Planning for steerable bevel-tip needle insertion through 2D soft tissue with obstacles," in Proc. IEEE Int. Conf. Robotics and Automation (ICRA), Apr. 2005 pp. 1652-1657.

[22] R. Alterovitz, T. Siméon, and K. Goldberg, "The Stochastic Motion Roadmap: A sampling framework for planning with Markov motion uncertainty," in Proc. Robotics: Science and Systems III, Jun. 2007.

[23] R. Alterovitz, M. Branicky, and K. Goldberg, "Motion planning under uncertainty for image-guided medical needle steering," Int. J. Robotics Research, vol. 27, no. 11-12, pp. 1361-1374, Nov. 2008.

[24] V. Duindam, J. Xu, R. Alterovitz, S. Sastry, and K. Goldberg, "Threedimensional motion planning algorithms for steerable needles using inverse kinematics," Int. J. Robotics Research, vol. 29, no. 7, pp. 789800, Jun. 2010.

[25] W. Park, Y. Wang, and G. S. Chirikjian, "The path-of-probability algorithm for steering and feedback control of flexible needles," Int. J. Robotics Research, vol. 29, no. 7, pp. 813-830, Jun. 2010

[26] J. van den Berg, S. Patil, R. Alterovitz, P. Abbeel, and K. Goldberg, "LQG-based planning, sensing, and control of steerable needles," in in Algorithmic Foundations of Robotics IX (Proc. WAFR 2010), ser Springer Tracts in Advanced Robotics (STAR), D. Hsu et al., Eds., vol. 68. Springer, Dec. 2010, pp. 373-389.

[27] J. van den Berg, P. Abbeel, and K. Goldberg, "LQG-MP: Optimized path planning for robots with motion uncertainty and imperfect state information," in Proc. Robotics: Science and Systems, June 2010.

[28] E. W. Stacy and G. A. Mihram, "Parameter estimation for a generalized gamma distribution," Technometrics, vol. 7, no. 3, pp. 349-358, 1965.

[29] E. Larsen, S. Gottschalk, M. C. Lin, and D. Manocha, "Fast proximity queries with swept sphere volumes," in Proc. IEEE Int. Conf. Robotics and Automation (ICRA), Apr. 2000, pp. 3719-3726. 